Research Paper

\title{
Subtyping Of Triple Negative Breast Carcinoma On The Basis Of RTK Expression
}

\author{
Harald Hessel1, Manuela Poignée-Heger 2 , Sabine Lohmann², Bianca Hirscher ${ }^{3}$, Andrea Herold ${ }^{2}$, Gerald \\ Assmann ${ }^{1,4}$, Jan Budczies ${ }^{5}$, Karl Sotlar ${ }^{1,6}$, Thomas Kirchner ${ }^{1}$ \\ 1. Institute of Pathology, Faculty of Medicine, LMU Munich, Germany \\ 2. Roche Diagnostics GmbH, Penzberg, Germany \\ 3. Roche Diagnostics International AG, Rotkreuz, Switzerland \\ 4. Pathologiepraxis München, Germany \\ 5. Institute of Pathology, Charité University Hospital, Berlin, Germany \\ 6. University Institute of Pathology, University Hospital Salzburg, Paracelsus Medical University, Austria \\ $\triangle$ Corresponding author: Dr. Harald Hessel, Institute of Pathology, Faculty of Medicine, LMU Munich, Thalkirchner Strasse 36,80337 München, Germany. \\ harald.hessel@med.uni-muenchen.de; Phone: +49 892180 73680; Fax: +4989 218073742 \\ () Ivyspring International Publisher. This is an open access article distributed under the terms of the Creative Commons Attribution (CC BY-NC) license \\ (https://creativecommons.org/licenses/by-nc/4.0/). See http://ivyspring.com/terms for full terms and conditions.
}

Received: 2017.09.26; Accepted: 2018.04.16; Published: 2018.06.23

\begin{abstract}
Background: "Triple-negative breast cancers" (TNBC) comprise a heterogeneous group of about $15 \%$ of invasive BCs lacking the expression of estrogen and progesterone receptors (ER, PR) and the expression of HER2 (ERBB2) and are therefore no established candidates for targeted treatment options in BC, i.e., endocrine and anti-HER2 therapy. The aim of the present study was to use gene expression profiling and immunohistochemical (IHC) characterization to identify receptor tyrosine kinase (RTK) profiles that would allow patient stratification for the purposes of target-oriented personalized tumor therapy in TNBC.

Methods: Twenty-nine cases of TNBC selected according to routine diagnostic IHC/cytogenetic criteria were examined by reverse transcription polymerase chain reaction (RT-PCR). RTK mRNA expression profiles were generated for a total of 31 tumor-relevant biomarkers, mainly belonging to the IGF- and EGF-receptor families but also including biomarkers related to downstream signaling. Protein expression of selected biomarkers was investigated by IHC.

Results: Hierarchical cluster analysis revealed a dichotomous differentiation pattern amongst TNBCs. A significant difference in gene expression was observed for 16 of the 31 RTK-associated tumor relevant biomarkers between the two newly identified TNBC subgroups. The findings were verified at the posttranslational level by the IHC data. The RTKs HER4, IGF-IR and IGF-2R and the hormone receptors ER and PR below the IHC detection limit play a central role in the differentiation of the two TNBC subgroups. Observed survival was reported as Kaplan-Meier estimates and point towards an improved survival of patients with RTK-high with superior three-year survival rate of $100 \%$ compared to RTK-low gene signatures with superior three-year survival rate of $60 \%$ (log-rank test, $\mathrm{p}$-value $=0.022$ ).

Conclusion: Gene-expression and IHC analysis of the EGF and IGF receptor families and biomarkers associated with downstream signaling point to the existence of two distinct TNBC subtypes. The RTKs HER4, IGF-1R, IGF-2R and the hormone receptors ER and PR appear to be of particular importance here. Based on survival analysis the differentiation of TNBC with RTK-high and RTK-low gene signatures seems to be of prognostic relevance. Additionally, correlation analysis of the relationship between RTKs and ER suggests co-regulatory mechanisms that may have potential significance in new therapeutic approaches.
\end{abstract}

Key words: triple-negative breast cancer, subtyping, personalized tumor therapy, Real-Time RT-PCR, receptor tyrosine kinase (RTK), RTK-high gene signature, RTK-low gene signature

\section{Introduction}

About one in eight women will be diagnosed with breast cancer during their lifetime, making this the most frequent malignant diseases in women [1].
Figures published by the WHO indicate that, worldwide, about 1,000,000 women develop breast cancer every year. Breast cancer comprises a 
heterogeneous group of malignant diseases that can be differentiated primarily on the basis of histopathological criteria into several distinct sub-groups of varying incidences [2].

Intense research efforts in recent years have provided insight into a large number of the complex molecular mechanisms involved in the pathogenesis of breast cancer [3]. Current attempts to develop individualized tumor therapies are aimed at identifying and treating further distinct subtypes on the basis of therapeutically relevant surrogate markers [4, 5, 6, 7, 8]. In addition to the classical histopathological parameters, the IHC characterization of hormone receptor status and HER2 as well as when indicated the cytogenetic detection of HER2 gene amplification represent the first important parameters upon which individualized tumor-specific therapy for breast carcinoma can be based. About $85 \%$ of all breast cancer patients currently receive such individualized therapy, which is aimed at the inhibition of HER2 (Trastuzumab, Lapatinib) and estrogen receptors (Tamoxifen, Letrozol) and is now considered standard treatment [9].

Thus, breast carcinoma can be roughly divided into tumors that are positive for estrogen/progesterone receptors and/or HER2 (triple-positive) which are suited to established, personalized therapy and those that are negative for these markers (triple-negative) without such benefit.

Triple-negative tumors are primarily defined, therefore, by an absence of surrogate markers of relevance for individualized therapy. As has been shown in numerous studies, triple-negative tumors vary in tumor progression, the development of therapeutic resistance, and the clinical course $[10,11$, $12,13,14,15]$, which indicates that they comprise a heterogeneous group. Further characterization and subtyping of triple-negative tumors on the basis of therapeutically relevant biomarkers is therefore strongly necessary.

To this end, a number of international microarray and NGS studies have produced data on the characterization of triple-negative tumors at the molecular level [13, 16, 17, 18]. In addition, further attempts to subtype these tumors have differentiated between basal-like and myoepithelial tumors $[19,20$, 21] the findings being primarily of prognostic relevance. However, little therapeutic strategies targeting basal-like tumor cell specific surrogate markers have yet been developed.

The search for new approaches to individualized therapy during recent years has revealed biomarkers with predictive relevance for a large number of malignant diseases, which have been used in the development of individualized tumor therapy modalities in clinical trials. Various RTK families are of particular importance here. The treatment success achieved with trastuzumab (Herceptin) in HER2+ breast cancer has stimulated a search for other therapeutic targets including other members of elements of the epidermal growth factor receptor (EGFR) family.

The concept of growth factor receptor based anti-tumor therapy has been investigated and developed in recent years, especially in relation to EGFR. This has produced a series of EGFR inhibitors that have been approved for the treatment of epithelial tumors. In addition to the therapeutic monoclonal antibodies cetuximab and panitumumab, which are of great importance in the treatment of colorectal carcinoma, antineoplastic effects have been demonstrated for the receptor tyrosine kinase inhibitors (RTKI) gefitinib (Iressa) and erlotinib (Rarceva) in non-small cell lung cancer and for lapatinib (Tykerb) in metastatic breast cancer [22, 23, 24].

In addition to the representatives of the EGF receptor family, insulin-like growth factor receptor 1 (IGF-1R) is a further promising candidate for the development of individual therapeutic approaches in oncology. Various specific therapeutic monoclonal antibodies and RTKI against IGF-1R, growth factor antagonists and IGF-1R are currently undergoing clinical trials $[25,26,27]$.

The aim of the present study was, by means of gene expression analysis and IHC investigations, to identify various RTK of the EGFR and IGFR families that would enable patient stratification for the purposes of target-oriented individualized tumor therapy in TNBC. In addition, correlation analysis was undertaken to shed light on possible interactions amongst the various RTK and provide a basis for innovative approaches to individualized tumor therapy.

\section{Materials and Methods}

\section{Patients and tumors}

A total of 29 triple-negative invasive ductal breast carcinomas, which had been diagnosed in the course of routine histopathological investigation at the Institute of Pathology, Ludwig-MaximiliansUniversität Munich during the years 2003 to 2005, were selected for investigation. All tumors were re-determined for negative hormone receptor and negative HER2 status according to up-to-date histopathological guidelines and recommendations $[28,29]$. ER and PR negativity was defined as positive staining in $<1 \%$ of tumor cells. HER2 negativity was 
defined as an IHC score of 0 or 1+; those with an immunoreactive score of $2+$ underwent HER2-specific FISH analysis to exclude HER2 gene amplification. Further selection criteria included the high-risk parameters of high tumor grade (grade 3 ) and low age (cut-off of $\leq 55$ years) at primary manifestation of disease (average age 41 years; $x=41.3$ years; $s=7.8$ years) at the time of diagnosis. Tumor size was documented in relation to the patient specific chemotherapeutic treatment regimen before adjuvant respectively before neoadjuvant chemotherapeutic treatment. The mean tumor size was $3.3 \mathrm{~cm}(x=3.3$ $\mathrm{cm}, \mathrm{s}=3.5 \mathrm{~cm}$ ). Nineteen patients received adjuvant chemotherapy and 9 patients neoadjuvant chemotherapy. Within the observation time of this study recurrence rate was $24 \%$ for patients treated with adjuvant chemotherapy and $63 \%$ for patients with neoadjuvant treatment. None of the patiens with neoadjuvant chemotherapy showed a pathological complete response (pCR). Tumors in 6 patients showed an infiltration of lymph or blood vessels (lymphangiosis-, hemangiosis carcinomatosa) at the time of diagnosis and one patient had distant metastases. Details of the diagnostically relevant parameters in the study group are given in Table 1.

\section{Material investigated}

The investigations were performed on formalin-fixed, paraffin-embedded tissue (FFPET) processed for routine histopathological diagnostic investigation. All analyses were performed at resection specimens.

\section{Deparaffinisation and RNA extraction}

For each case, nine 4- $\mu \mathrm{m}$ FFPET whole sections comprising more than $70 \%$ tumor were cut and deparaffinized according to standard protocols. Total RNA was isolated by means of a commercial RNA isolation kit (High-Pure RNA Isolation Kit, Roche). RNA samples were analyzed photometric for their qualitative and quantitative content (NanoDrop, Thermo Scientific, USA). The minimum RNA content required for gene expression analysis was $300 \mathrm{ng} / \mu \mathrm{l}$ (total volume: $45 \mu \mathrm{l}$ ). An adequate amount of total RNA could be isolated from a total of 29 of the 31 triple-negative tumors that were initially selected for investigation.

\section{cDNA synthesis}

cDNA synthesis was performed by primer-specific reverse transcription of total RNA according to a standard protocol (Transkriptor First Strand cDNA Synthesis Kit, Roche). In brief, $3 \mu 1$ RNA $(50 \mathrm{ng} / \mu \mathrm{l}), 1 \mu \mathrm{l}$ sequence-specific primer $(1 \mu \mathrm{M}), 9 \mu \mathrm{l}$ distilled water, $4 \mu \mathrm{l} 5 \mathrm{X}$ buffer, $2 \mu \mathrm{l}$ nucleotide mix (each $10 \mathrm{mM}$ ), $0.5 \mu \mathrm{l}$ protector RNase inhibitor $(40$ $\mathrm{U} / \mu \mathrm{l})$ and $0.5 \mu \mathrm{l}$ transcriptor reverse transcriptase (20 $\mathrm{U} / \mu \mathrm{l})$ per reaction were mixed, preincubated for 10 minutes at $95^{\circ} \mathrm{C}$ and incubated for one hour at $50^{\circ} \mathrm{C}$.

Table 1. Diagnostically relevant parameters in the study group

\begin{tabular}{|c|c|c|c|c|c|c|c|c|c|c|c|}
\hline Case & Age & Grade & Estrogen & Progesterone & $\begin{array}{l}\text { HER2/ } \\
\text { neu }\end{array}$ & $\begin{array}{l}\text { Lymph } \\
\text { nodes }\end{array}$ & $\begin{array}{l}\text { Tumor } \\
\text { size }(\mathrm{cm})\end{array}$ & $\begin{array}{l}\text { Lymphangiosis carcinomatosa / } \\
\text { Angiosis carcinomatosa }\end{array}$ & $\begin{array}{l}\text { Neoadjuvant } \\
\text { chemo-therapy }\end{array}$ & $\begin{array}{l}\text { Adjuvant } \\
\text { chemo-therapy }\end{array}$ & $\begin{array}{l}\text { Distant } \\
\text { metastases }\end{array}$ \\
\hline 1 & 42 & 3 & 0 & 0 & 0 & 0 & 2.3 & & & $\mathrm{x}$ & \\
\hline 2 & 31 & 3 & 0 & 0 & $1+$ & 1 & 1.6 & $x$ & $x$ & & \\
\hline 3 & 43 & 3 & 0 & 0 & 0 & 1 & 1.5 & & & $\mathrm{x}$ & \\
\hline 4 & 42 & 3 & 0 & 0 & 0 & 0 & 1.1 & & & $\mathrm{x}$ & \\
\hline 5 & 53 & 3 & 0 & 0 & 0 & 0 & 1.3 & & & $\mathrm{x}$ & \\
\hline 6 & 38 & 3 & 0 & 0 & $1+$ & 0 & 1.6 & & & $x$ & \\
\hline 7 & 45 & 3 & 0 & 0 & 0 & 1 & 15 & $\mathrm{x}$ & & $\mathrm{x}$ & \\
\hline 8 & 48 & 3 & 0 & 0 & $1+$ & 0 & 2.5 & $x$ & $x$ & & \\
\hline 9 & 28 & 3 & 0 & 0 & $1+$ & 0 & 1.9 & & & $x$ & \\
\hline 10 & 42 & 3 & 0 & 0 & 0 & 0 & nd & $x$ & $x$ & & \\
\hline 11 & 34 & 3 & 0 & 0 & 0 & nd & 3.3 & & $x$ & & \\
\hline 12 & 31 & 3 & 0 & 0 & 0 & 1 & 3.6 & & $x$ & & \\
\hline 13 & 47 & 3 & 0 & 0 & 0 & nd & nd & & nd & nd & \\
\hline 14 & 53 & 3 & 0 & 0 & 0 & 0 & 4.3 & & & $x$ & \\
\hline 15 & 34 & 3 & 0 & 0 & 0 & 1 & 2.2 & & & $x$ & \\
\hline 16 & 51 & 3 & 0 & 0 & 0 & 0 & 0.7 & & & $x$ & \\
\hline 17 & 40 & 3 & 0 & 0 & 0 & 0 & 2.2 & & & $x$ & \\
\hline 18 & 49 & 3 & 0 & 0 & 0 & nd & 0.9 & & & $x$ & \\
\hline 19 & 53 & 3 & 0 & 0 & 0 & 1 & 2.5 & & $x$ & & $x$ \\
\hline 20 & 51 & 3 & 0 & 0 & $2+$ & 0 & 5 & & $x$ & & \\
\hline 21 & 35 & 3 & 0 & 0 & $2+$ & 1 & 3 & $x$ & $x$ & & \\
\hline 22 & 49 & 3 & 0 & 0 & $1+$ & 0 & 2.1 & & & $x$ & \\
\hline 23 & 43 & 3 & 0 & 0 & $1+$ & 1 & 2 & $x$ & & $x$ & \\
\hline 24 & 35 & 3 & 0 & 0 & 0 & 0 & 2 & & $x$ & & \\
\hline 25 & 33 & 3 & 0 & 0 & $2+$ & 0 & 2.6 & & & $x$ & \\
\hline 26 & 41 & 3 & 0 & 0 & $1+$ & 0 & 10 & & & $x$ & \\
\hline 27 & 32 & 3 & 0 & 0 & 0 & 0 & 2.5 & & & $x$ & \\
\hline 28 & 30 & 3 & 0 & 0 & 0 & 1 & 2 & & & $x$ & \\
\hline 29 & 46 & 3 & 0 & 0 & 0 & 0 & 5 & & & $x$ & \\
\hline
\end{tabular}


Per 96-well plate a standard dilution series (dilution factor 1:5), calibrator, negative control and 29 tumor samples were processed. A total of 34 biomarkers, including representatives of the EGFR and IGF-1R families and biomarkers related to downstream signaling, and five housekeeping genes (HKG) were analyzed in separate runs.

\section{PCR Amplification}

Gene expression data were obtained using sequence-specific detection probes (hydrolysis probes). With the help of the Universal ProbeLibrary Software [30], primer-specific sequences and target-specific hydrolysis probes for 34 biomarkers and 5 HKG were determined on the basis of freely accessible data banks (Table 2 ).

Table 2. Biomarkers / Universal ProbeLibrary Assays

\begin{tabular}{|c|c|c|c|}
\hline Biomarker & Primersequence (5' -> 3'-direction) & Probe No. & Reverse Primer RT \\
\hline EGFR Fwd & cat gtc gat gga ctt cca ga & 44 & ttc tcc tct cet gca cc \\
\hline EGFR Rev1 & ggg aca get tgg atc aca ct & & \\
\hline HER2 Fwd & tgg ctc agt gac ctg ttt tg & 75 & tca ggt ttc aca ccg ct \\
\hline HER2 Rev1 & ggt cct tat agt ggg cac agg & & \\
\hline Her3 Fwd & cac aat gcc gac ctc tcc & 86 & tgg gca atg gta gag tag ag \\
\hline Her3 Rev1 & cac gag gac ata gcc tgt ca & & \\
\hline Her4 Fwd & ttc cac ttt acc aca aca tgc ta & 78 & aca gca aat gtc aga ccc \\
\hline Her4 Rev1 & cag aat gaa gag ccc acc a & & \\
\hline TGF-alpha Fwd & $\operatorname{tgc} \operatorname{tgc} c a c t c a$ gaa aca gt & 63 & agt gct gtc ctg aag aag c \\
\hline TGF-alpha Rev1 & atc tgc cac ag tcc acc tg & & \\
\hline EGF Fwd & $\operatorname{tgg} \operatorname{ttg} \operatorname{tgg}$ ttc atc cat tg & 68 & tca cag cct ccg ttt tga ta \\
\hline EGF Rev1 & tca cag cct ccg ttt tga ta & & \\
\hline beta-cellulin Fwd & act gca tca aag gga gat gc & 49 & acc ttg ctc caa tgt agc \\
\hline beta-cellulin Rev1 & tcc aat gta gcc ttc atc aca & & \\
\hline Amphiregulin Fwd & cgg aga atg caa ata tat aga gca c & 38 & tgt caa tca tgc tgt gag ttt \\
\hline Amphiregulin Rev1 & cac cga aat att ctt gct gac a & & \\
\hline HB-EGF Fwd & tgg ggc ttc tca tgt tta gg & 55 & ccg att cct tga gca ca \\
\hline HB-EGF Rev1 & tgc cca act tca ctt tct ctt c & & \\
\hline NRG-Common Fwd & gat cag caa att agg aaa tga cag & 53 & tct gaa gac aca tat get cct \\
\hline NRG-Common Rev1 & ggc ata cca gtg atg atc tcg & & \\
\hline NRG-GGF2 Fwd & get gcc act act gct gct g & 70 & cgg gga cga gta gca cac \\
\hline NRG-GGF2 Rev1 & cgg gga cga gta gca cac & & \\
\hline NRG-SMDFsecSet Fwd & aga acg ccc aag tca gca & 5 & atg get tgt ccc agt $g$ \\
\hline NRG-SMDF secSetRev1 & ttg tcc cag tgg tgg atg ta & & \\
\hline NRG2 Fwd & ttc gca tca aat atg gca ac & 39 & gac ggt gtc ctt $\operatorname{ccc} c$ \\
\hline NRG2 Rev1 & ggc ctc gca gac ata ctc $c$ & & \\
\hline NRG3 Fwd & gcc agt ctg tca aac acc ac & 26 & cca cct agc cta ctt cgg \\
\hline NRG3 Rev1 & atg gag cat gcc act tct tt & & \\
\hline NRG4 Fwd & ggg gct ttg tta tgt gat acc $t$ & 23 & ctg gag cct ggg aga a \\
\hline NRG4 Rev1 & cct gta tag ttt tca acg cac ct & & \\
\hline BCL2 Fwd & agg tgc atc tgg tga tgt ga & 26 & cac tcc aac ccc cga tct \\
\hline BCL2 Rev1 & cac tcc aac ccc cga tct & & \\
\hline G6PDH secSet Fwd & aga gac cgt gga tgc tga ag & 65 & ctc cgc act get gac a \\
\hline G6PDH secSet Rev1 & tga gga cct ccg tca gat g & & \\
\hline beta-Aktin Fwd & att ggc aat gag cgg ttc & 11 & atg tcc acg tca cac ttc at \\
\hline beta-Atkin Rev & gga tgc cac agg act cca $t$ & & \\
\hline ER-Fwd & aac cag tgc acc att gat aaa a & 69 & att ctc cet cet ctt cgg \\
\hline ER-Rev & tcc tct tcg gtc ttt tcg tat c & & \\
\hline PGR-Fwd & ttt aag agg gca atg gaa gg & 11 & $\mathrm{ttt} t \mathrm{tc}$ tgc gga $\mathrm{ttt}$ tat caa \\
\hline PGR-Rev & cgg att tta tca acg atg cag & & \\
\hline PTEN-Fwd & gca caa gag gcc cta gat ttc & 60 & aat aca cat agc gec tct ga \\
\hline PTEN-Rev & cgc ctc tga ctg gga ata gt & & \\
\hline PIK3CA-Fwd & cga gat cct ctc tct gaa atc ac & 2 & gaa ttt cgg gga tag tta cac aa \\
\hline PIK3CA-Rev & gaa ttt cgg gga tag tta cac aa & & \\
\hline PIK3R1 Iso 1-Fwd & aat gaa cga cag cct gca c & 16 & cag tac cat tca gca tct tgt aa \\
\hline PIK3R1 Iso 1-Rev & ccg ttg ttg gct aca gta gta gg & & \\
\hline AKT common-Fwd & ggc tca ccc agt gac aac tc & 41 & agc agc ttc agg tac tca aa \\
\hline AKT common-Rev & act caa act cgt tca tgg tca $c$ & & \\
\hline SRCcom-Fwd & cga gaa agt gag acc acg aa & 34 & gtt gag gcc ctt ggc \\
\hline SRCcom-Rev & ttg gcg ttg tcg aag tca & & \\
\hline SCUBE2-Fwd & get gec atc cac agt aca ag & 17 & gac agt gtc ctc tcg ctc caa \\
\hline SCUBE2-Rev & gac agt gtc ctc tcg ctc caa & & \\
\hline Ki-67-Fwd & tga att tcc aag aaa aat acg tga & 36 & agc ttt ctc atc agg gtc a \\
\hline Ki-67-Rev & tca ggg tca gaa gag aag cta ga & & \\
\hline Cyclin B1-Fwd & $\operatorname{cgc} \operatorname{ctg}$ agc cta ttt tgg t & 34 & aga aag cct gac aca ggt $c$ \\
\hline Cyclin B1-Rev & gca cat cca gat gtt tcc att & & \\
\hline HNF3a-Fwd & agg gct gga tgg ttg tat tg & 1 & atg ttg ctg acc ggg a \\
\hline HNF3a-Rev & acc ggg acg gag gag tag & & \\
\hline
\end{tabular}




\begin{tabular}{|c|c|c|c|}
\hline Biomarker & Primersequence (5' -> 3'-direction) & Probe No. & Reverse Primer RT \\
\hline Survivin com-Fwd & ctt gaa agt ggc acc aga gg & 29 & cct cac ttc tca cct ggt aa \\
\hline Survivin com-Rev & caa aaa tga gcc ccc aaa a & & \\
\hline TRIP13-Fwd & ctc atg cgc tgt atg tcc a & 65 & gca agc ttc ttt ctc tct tca \\
\hline TRIP13-Rev & gtc cac tgc cag aga cag g & & \\
\hline HPRT-Fwd & tga cct tga ttt att ttg cat acc & 73 & aat gtg atg gcc tcc ca \\
\hline HPRT-Rev & cga gca aga cgt tca gtc ct & & \\
\hline PBGD common-Fwd & agc tat gaa gga tgg gca ac & 25 & gcc tgc atg gtc tct tgt a \\
\hline PBGD common-Rev & ttg tat gct atc tga gcc gtc ta & & \\
\hline PPP1CA common-Fwd & cct ata aga tca agt acc ccg aga & 15 & cag ttg aag cag tca gtg aa \\
\hline PPP1CA common-Rev & gat gtt gta gcg tct ctt gca c & & \\
\hline IGF1 Fwd & tgt gga gac agg ggc ttt ta & 67 & cac tca tcc acg atg cct \\
\hline IGF1 Rev & atc cac gat gec tgt ctg a & & \\
\hline IGF2 Fwd & gct ggc aga gga gtg tcc & 10 & cat tgg gat tcc cat tgg \\
\hline IGF2 Rev & gat tcc cat tgg tgt ctg ga & & \\
\hline IGF-1R Fwd & ttc agc get get gat gtg & 10 & caa gtt ccc gge tca tg \\
\hline IGF-1R Rev & ggc tca tgg tga tct tct $c c$ & & \\
\hline IGF-2R Fwd & cag acc aca tat acc acg agg a & 1 & agt tga gag aaa aga tgg ggt \\
\hline IGF-2R Rev & aga aaa gat ggg gtg gct gt & & \\
\hline
\end{tabular}

Table 3. Antibodies employed in the study

\begin{tabular}{|c|c|c|c|c|c|c|}
\hline Antigen & Source & Order No. & Dilution & Method / System & Pretreatment & Incubation \\
\hline EGFR & Ventana/Roche & $790-2988$ & ready to use & Ventana /3,3'-diamino-benzidine kit ${ }^{1}$ & 20 min, Protease & $32 \mathrm{~min}$ \\
\hline HER2 & Ventana/Roche & $800-2996$ & ready to use & Ventana /3,3-diamino-benzidine kit ${ }^{1}$ & 30min, CC1 & $16 \mathrm{~min}$ \\
\hline IGF-1R & Ventana/Roche & $790-4346$ & ready to use & Ventana /3,3'-diamino-benzidine kit ${ }^{1}$ & $60 \mathrm{~min}, \mathrm{CC} 1$ & $28 \mathrm{~min}$ \\
\hline ER & Ventana/Roche & $790-4325$ & ready to use & Ventana /3,3-diamino-benzidine kit ${ }^{1}$ & $60 \mathrm{~min}, \mathrm{CC} 1$ & $28 \mathrm{~min}$ \\
\hline PR & Ventana/Roche & $790-4296$ & ready to use & Ventana /3,3'-diamino-benzidine kit ${ }^{1}$ & $60 \mathrm{~min}, \mathrm{CC} 1$ & $28 \mathrm{~min}$ \\
\hline CK $5 / 6$ & Ventana/Roche & $760-4253$ & ready to use & Ventana /3,3-diamino-benzidine kit ${ }^{1}$ & $60 \mathrm{~min}, \mathrm{CC} 1$ & $32 \mathrm{~min}$ \\
\hline
\end{tabular}

${ }^{1}$ (XT Ultra View DAB kit, Ventana Medical Systems, Tucson, Arizona)

For quantitative PCR, $3.5 \mu \mathrm{l}$ cDNA $(50 \mathrm{ng} / \mu \mathrm{l})$ were added to $16.5 \mu \mathrm{l} \mathrm{PCR}$ Master Mix according to the manufacturer's instructions (LightCycler ${ }^{\circledR} 480$ Probes Master, Roche). For all targets, uniform ThermoCycler running conditions were used. In brief, initial preincubation at $95^{\circ} \mathrm{C}$ for 5 minutes was followed by 45 cycles of $95^{\circ} \mathrm{C}$ for 10 seconds, $60^{\circ} \mathrm{C}$ for 20 seconds and $72^{\circ} \mathrm{C}$ for 1 second. Gene expression profiles were generated on the LightCycler ${ }^{\circledR} 480$ Real-Time PCR System (Roche).

A standard curve, consisting of diluted human reference RNA (Universal Human Reference RNA, Stratagene), was included for every RT reaction and subsequent RT-PCR. For a run to be accepted there had to be a valid negative control and an amplification efficiency of between 1.9 and 2.1. Expression data were normalized to five averaged HKG. All experiments were performed in duplicate and the results averaged. Calibrator based calculations were used to determine relative gene expression profiles [31].

\section{Immunohistochemical analysis}

IHC investigation for various surrogate markers was performed to substantiate the results of gene expression analysis and to identify basal-like breast carcinomas. Markers investigated included the RTK EGFR, HER2 and IGF-1R, the hormone receptors ER and PR, and the basal-like marker CK5/6 [32]. Whole FFPET sections cut at $3 \mu \mathrm{m}$ were stained with a Ventana Benchmark XT autostainer (Ventana Medical
Systems). Details of the antibodies and methods employed are given in Table 3.

Slides were counterstained with haematoxylin (Ventana). System and isotype controls were included. IHC investigation for ER and PR and for HER2 was performed according to up-to-date histopathological guidelines and recommendations $[28,29]$.

Immunostaining for EGFR and IGF-1R was evaluated semiquantitatively with a scoring system similar to that established for HER2 (0: no membrane-specific staining, 1+: weak, incomplete cell membrane staining in $<10 \%$ of cells, $2+$ : weak or moderate staining of the complete cell membrane in $>$ $10 \%$ of cells, $3+:$ strong, complete membrane staining in $>10 \%$ of cells. Immunostaining for CK 5/6 was evaluated according to the criteria of Dabbs et al. [33] (0: no staining, R: single cells stained, $1+: 5-30 \%$ of cells stained, $2+:>30-60 \%$ of cells stained, $3+:>60 \%$ of cells stained). The scores 0 and $\mathrm{R}$ were considered negative, $1+$ to $3+$ positive.

\section{Statistical analysis}

Statistical evaluation was performed with the help of the statistics software SPSS (Chicago, USA) and R [34]. The Kolmogorov Smirnov test was used to test for normal distribution. The Mann-Whitney U test and Student's t-test were used to test for significant differences in gene expression between the postulated subtypes RTK-high and RTK-low. T-test for equality of means and Levene's test for equality of variances 
was applied to demonstrate the statistic independence of tumor samples with adjuvant and neoadjuvant chemotherapeutic treatment in context to the gene-expression analyses. The influence of clinical parameters on the gene expression profile was investigated by multivariate analysis on the basis of the Chi-square test and the Mann-Whitney U test. Correlation between variables was observed with Pearson respectively Spearman rank correlation analyses. Hierarchical cluster analysis was performed to identify subtypes within the study cohort. The 3 -year survival rate was analyzed with the Kaplan-Meier curve.

\section{Ethics approval and consent to participate}

Specimens and data were anonymized, and the need for consent was waived by the institutional ethics committee of the Medical Faculty of the Ludwig Maximilians University of Munich.

\section{Results}

\section{Biomarker detection efficiencies within RT-PCR analyses}

In the present study 34 tumor associated biomarkers relevant for EGFR and IGF-1R families and related biomarkers of the downstream signaling as well as 5 HKG were analyzed. Expression profiles for all tumor samples could be obtained for 31 of the biomarkers and all five HKG.

\section{Differentiation of triple-negative tumors into two distinct subtypes on the basis of gene expression profiling of RTK associated biomarkers}

Hierarchical cluster analysis revealed a dichotomous differentiation pattern amongst the triple-negative tumors (Figure 1).

The two subtypes were characterized by low (subtype RTK-low; $\mathrm{N}=15$ ) and high (subtype RTK-high; $\mathrm{N}=14$ ) gene expression of the biomarkers investigated. The corresponding relative gene expression values of each biomarker are shown in Table 4.

Further statistical analysis confirmed significant differences in gene expression between the subgroups RTK-low and RTK-high for 16 of the 31 biomarkers evaluated (Table 4). Of particular interest were significant differences for 3 of the 6 analyzed RTK (HER4, $\mathrm{p} \leq$ 0.001; IGF-1R, $\mathrm{p}<0,001$; IGF-2R, $\mathrm{p}=0.015$; Figure 2).

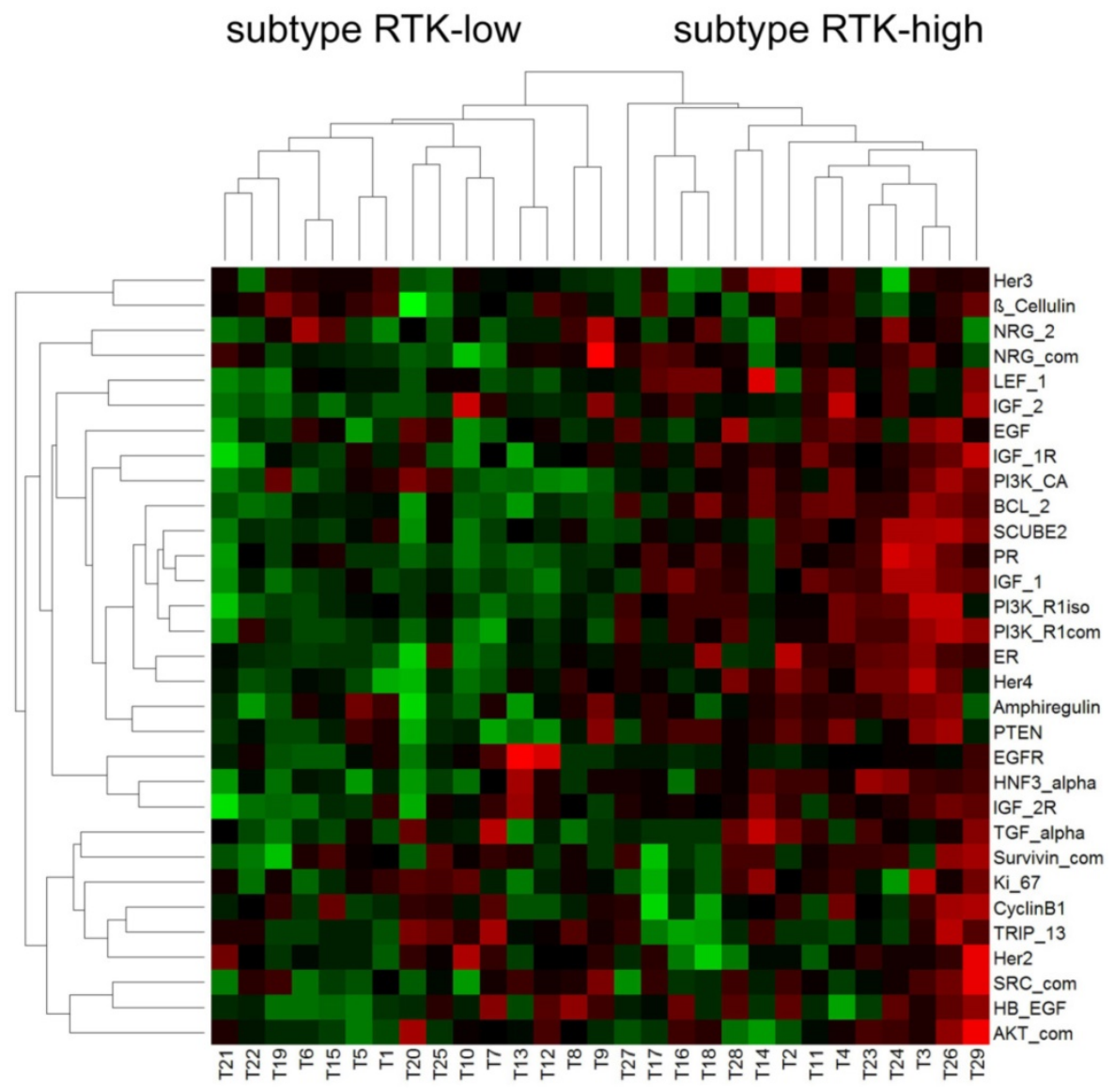

Figure 1. Pearson correlation - triple-negative breast cancer 
Table 4. Differential biomarker expression in triple-negative tumors of the subtypes RTK-low and RTK-high

\begin{tabular}{|c|c|c|c|c|c|c|c|c|c|}
\hline Biomarker & $\mathrm{p}$ & TNBC subtype & Mean & $95 \%$ CI & Biomarker & $\mathrm{p}$ & TNBC subtype & Mean & $95 \% \mathrm{CI}$ \\
\hline \multirow[t]{2}{*}{ PR } & 0.000 & RTK-low & 2.789 & $1.802-3.776$ & Amphiregulin & 0.24 & RTK-low & 2.880 & $0.840-4.920$ \\
\hline & & RTK-high & 17.582 & $5.256-29.908$ & & & RTK-high & 4.610 & $2.122-7.278$ \\
\hline \multirow[t]{2}{*}{ HER4 } & 0.000 & RTK-low & 1.510 & $0.827-2.373$ & NRG-com & 0.408 & RTK-low & 16.995 & $-16.498-50.488$ \\
\hline & & RTK-high & 10.550 & $3.887-17.213$ & & & RTK-high & 3.390 & $1.669-5.111$ \\
\hline \multirow[t]{2}{*}{ EGF } & 0.043 & RTK-low & 3.728 & $1.153-6.303$ & Ki-67 & 0.407 & RTK-low & 1.390 & $1.114-1.665$ \\
\hline & & RTK-high & 18.825 & $4.383-33.267$ & & & RTK-high & 1.628 & $1.069-2.186$ \\
\hline \multirow[t]{2}{*}{ PI3K-R1iso } & 0.000 & RTK-low & 4.494 & $3.659-5.329$ & SRC-com & 0.254 & RTK-low & 17.375 & $13.012-21.738$ \\
\hline & & RTK-high & 12.392 & 8.023 - 16.762 & & & RTK-high & 22.269 & 14.142 - 30.397 \\
\hline \multirow[t]{2}{*}{ SCUBE2 } & 0.003 & RTK-low & 5.696 & $3.953-7.439$ & Survivin-com & 0.119 & RTK-low & 0.262 & $0.185-0.339$ \\
\hline & & RTK-high & 22.497 & $9.788-35.206$ & & & RTK-high & 0.3981 & $0.228-0.568$ \\
\hline \multirow[t]{2}{*}{ BCL2 } & 0.000 & RTK-low & 10.077 & $7.813-12.340$ & TRIP13 & 0.382 & RTK-low & 1.214 & $0.948-1.479$ \\
\hline & & RTK-high & 34.702 & $26.705-42.699$ & & & RTK-high & 1.035 & $0.690-1.381$ \\
\hline \multirow[t]{2}{*}{ IGF-1R } & 0.001 & RTK-low & 1.424 & $0.876-1.971$ & Cyclin B1 & 0.425 & RTK-low & 0.92 & $0.738-1.102$ \\
\hline & & RTK-high & 4.332 & $2.159-6.504$ & & & RTK-high & 1.082 & $0.696-1.467$ \\
\hline \multirow[t]{2}{*}{ IGF-2R } & 0.015 & RTK-low & 2.545 & $1.827-3.263$ & HER2 & 0.794 & RTK-low & 6.353 & $5.173-7.533$ \\
\hline & & RTK-high & 3.493 & $2.980-4.007$ & & & RTK-high & 6.093 & $4.299-7.888$ \\
\hline \multirow[t]{2}{*}{ IGF2 } & 0.004 & RTK-low & 0.376 & $0.224-0.976$ & HER3 & 0.798 & RTK-low & 6.324 & $5.258-7.389$ \\
\hline & & RTK-high & 0.473 & $0.069-1.015$ & & & RTK-high & 8.650 & $5.070-12.231$ \\
\hline \multirow[t]{2}{*}{ HNF3-a } & 0.001 & RTK-low & 0.142 & $0.025-0.308$ & $\beta$-Cellulin & 0.889 & RTK-low & 63.354 & $37.120-89.588$ \\
\hline & & RTK-high & 0.306 & $0.135-0.477$ & & & RTK-high & 60.973 & $35.728-86.218$ \\
\hline \multirow[t]{2}{*}{ IGF1 } & 0.000 & RTK-low & 31.943 & $25.742-38.143$ & HBEGF & 0.446 & RTK-low & 3.408 & $1.849-4.968$ \\
\hline & & RTK-high & 113.493 & 73.942 - 153.044 & & & RTK-high & 4.189 & $2.689-5.688$ \\
\hline \multirow[t]{2}{*}{ ER } & 0.000 & RTK-low & 0.833 & $0.520-1.146$ & NRG-2 & 0.727 & RTK-low & 5.416 & $1.366-9.467$ \\
\hline & & RTK-high & 2.554 & $1.452-3.656$ & & & RTK-high & 4.651 & $2.572-6.730$ \\
\hline \multirow[t]{2}{*}{ PTEN } & 0.000 & RTK-low & 3.704 & $2.278-5.129$ & AKT-com & 0.311 & RTK-low & 4.606 & $3.578-5.633$ \\
\hline & & RTK-high & 6.786 & $4.883-8.689$ & & & RTK-high & 5.675 & $3.640-7.710$ \\
\hline \multirow[t]{2}{*}{ Pi3K-CA } & 0.043 & RTK-low & 5.415 & $3.521-7.310$ & EGFR & 0.257 & RTK-low & 23.781 & $-12.782-60.344$ \\
\hline & & RTK-high & 8.481 & $6.383-10.579$ & & & RTK-high & 3.645 & $2.668-4.621$ \\
\hline \multirow[t]{2}{*}{ LEF1 } & 0.003 & RTK-low & 0.871 & $0.684-0.106$ & TGF- $\alpha$ & 0.219 & RTK-low & 3.587 & $1.112-6.062$ \\
\hline & & RTK-high & 0.2561 & $0.131-0.381$ & & & RTK-high & 5.865 & $2.817-8.913$ \\
\hline \multirow[t]{2}{*}{ PI3K R1 iso } & 0.000 & RTK-low & 4.494 & $3.659-5.329$ & & & & & \\
\hline & & RTK-high & 12.392 & $8.023-16.762$ & & & & & \\
\hline
\end{tabular}

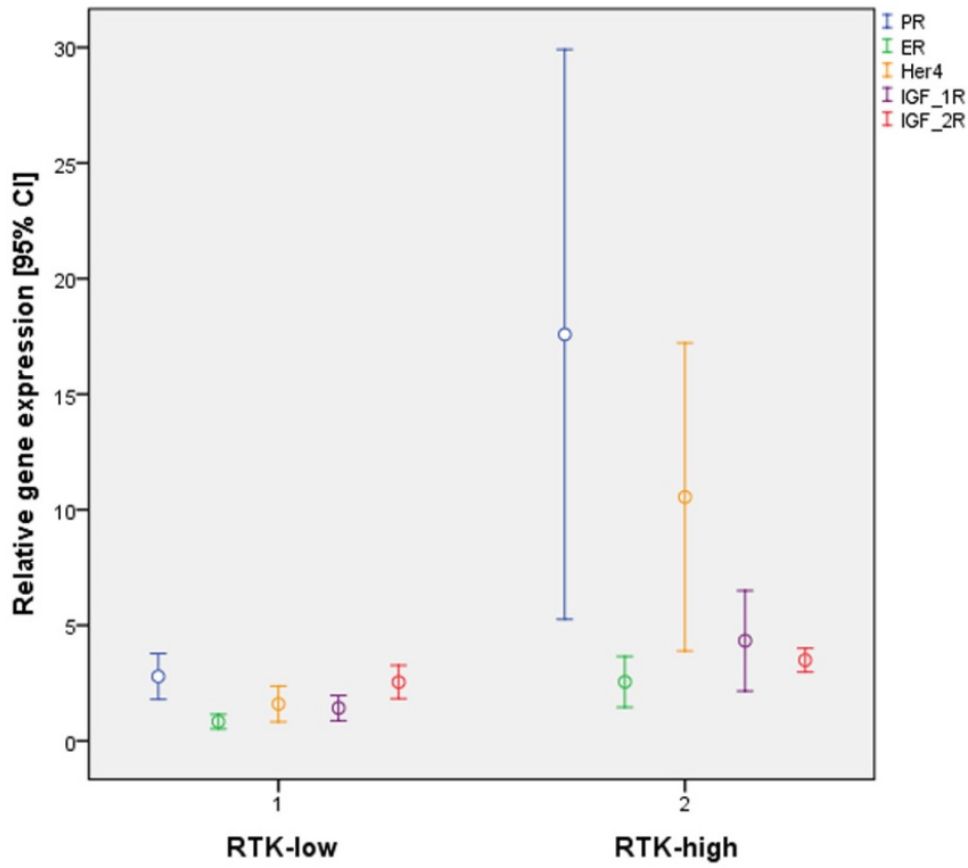

Figure 2. Differential gene expression by the triple-negative subtypes RTK-low und RTK-high in relation to PR, ER, HER4, IGF-1R and IGF-2R

Interestingly, significant differences in gene expression patterns for ER $(p=0.001)$ and PR $(p<$ 0.001) were also found between the two subtypes (Figure 2). By contrast, all cases of triple-negative tumors, by definition lacked IHC staining for ER and PR.
Correlation analysis was performed to determine whether the RTK and hormone receptors investigated are subject to functional interaction (Table 5). 
Table 5. Correlation coefficients for RTK, ER and PR gene expression

\begin{tabular}{lllllllll}
\hline & ER & PR & EGFR & egfr & HER3 & HER4 & IGF-1R IGF-2R \\
\hline ER & 1 & & & & & & & \\
PR & $0.654^{* *}$ & 1 & & & & & & \\
EGFR & 0.034 & 0.001 & 1 & & & & & \\
HER2 & 0.057 & -0.081 & 0.099 & 1 & & & & \\
HER3 & -0.109 & 0.078 & 0.023 & 0.000 & 1 & & & \\
HER4 & $0.675^{* *}$ & $0.623^{* *}$ & -0.021 & -0.105 & 0.222 & 1 & & \\
IGF-1R & $0.419^{*}$ & $0.555^{* *}$ & -0.056 & 0.112 & 0.206 & $0.394^{*}$ & 1 & \\
IGF-2R & 0.293 & 0.284 & $0.519^{* *}$ & -0.034 & 0.354 & $0.369^{*}$ & 0.264 & 1 \\
\hline${ }^{* *} \mathrm{p}<0.005 ;{ }^{*} \mathrm{p}<0.05$ & & & & & & & &
\end{tabular}

Increased correlation, in particular for HER4 with ER $(r=0.675 ; p \leq 0.001)$, HER4 with PR $(r=0.623$, $\mathrm{p} \leq 0.001)$, as well as IGF-1R with ER $(\mathrm{r}=0.419$; $\mathrm{p}=0.024)$ and IGF-1R with PR $(\mathrm{r}=0.555 ; \mathrm{p}=0.002)$ was found (Figure 3).

Additionally, we compared the patient survival based on the RTK-high and RTK-low gene signature. Kaplan-Meier analysis demonstrated superior three-year survival in the RTK-high group as opposed to the patients in the RTK-low group (three-year
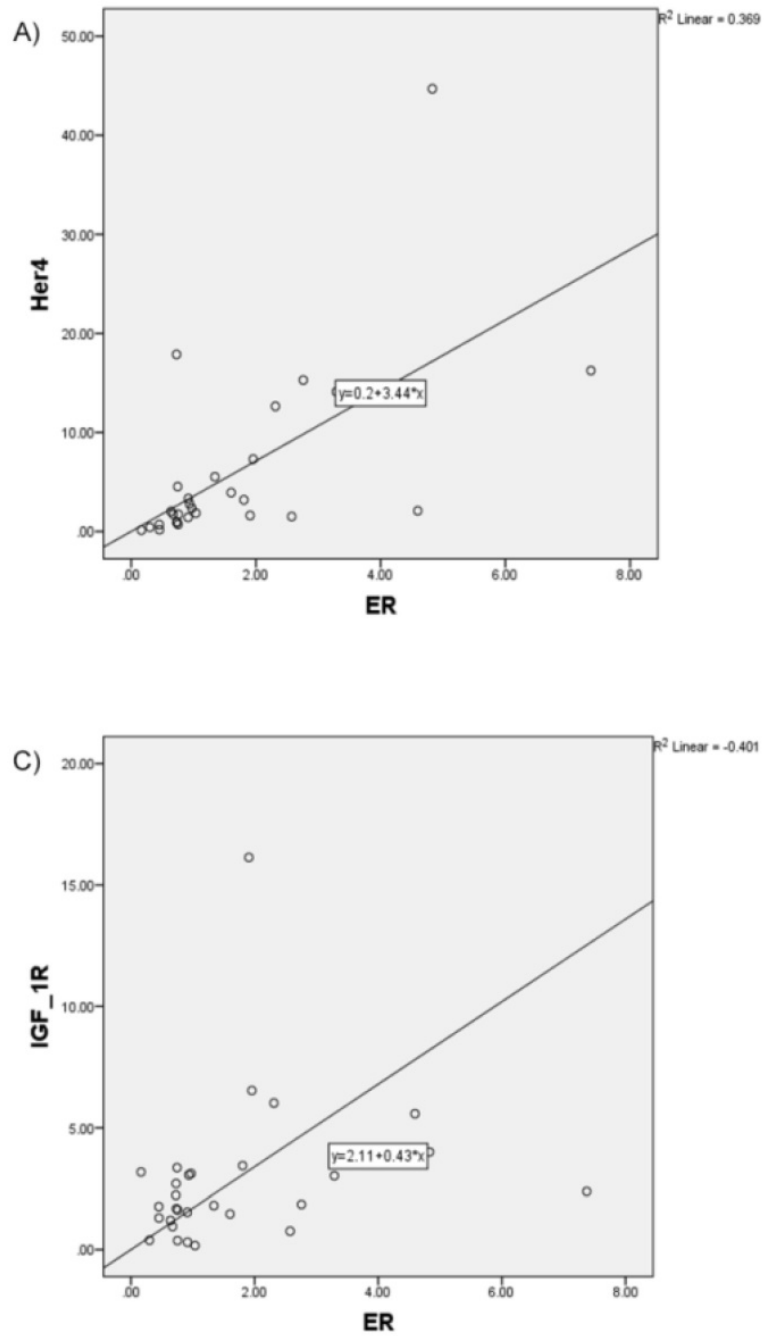

survival rate of $100 \%$ v. $60 \%$; log-rank test, p-value $=$ 0.022; Figure 4).

In summary, two subtypes of TNBC could be identified on the basis of gene expression profiles of 16 biomarkers of the EGF and IGF receptor families and biomarkers of their downstream signaling as well as the hormone receptors ER and PR (Table 4). Of special interest are the RTK HER4 and IGF-1R, as well as the hormone receptors ER and PR, for which the findings of correlation analysis indicate functional interaction in cell signaling.

\section{Comparison of IHC and gene expression findings}

In order to determine to what extent the differences in gene expression between the subtypes RTK-high and RTK-low as detected by RT-PCR are also reflected at the protein level, IHC investigations were carried out for certain key proteins from the biomarker panel (IGF-1R and EGFR: study findings, HER2, ER and PR: findings of routine diagnostic
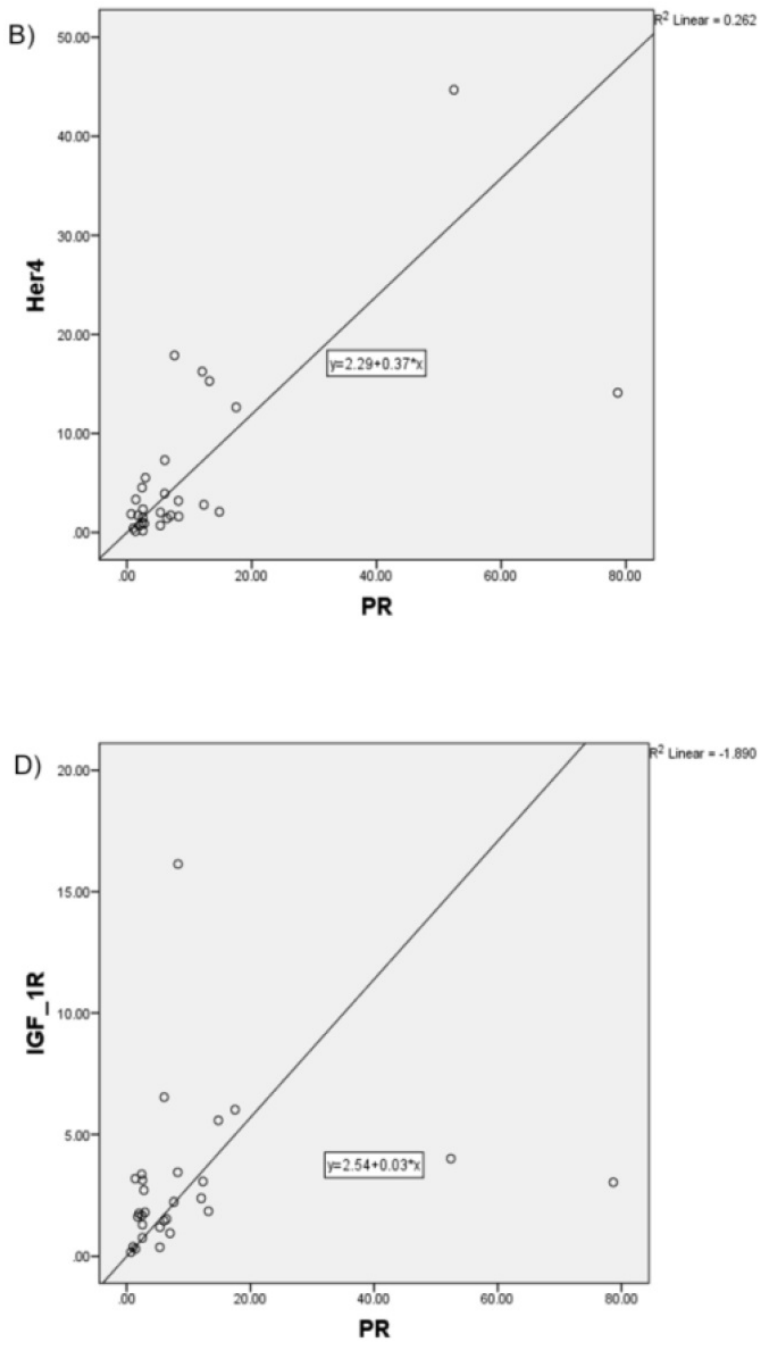

Figure 3. Increased correlation between analyzed RTK and hormone receptors pictured in scatter plot charts A) HER4 and ER, B) HER4 and PR, C) IGF-IR and ER, D) IGF-IR and PR 


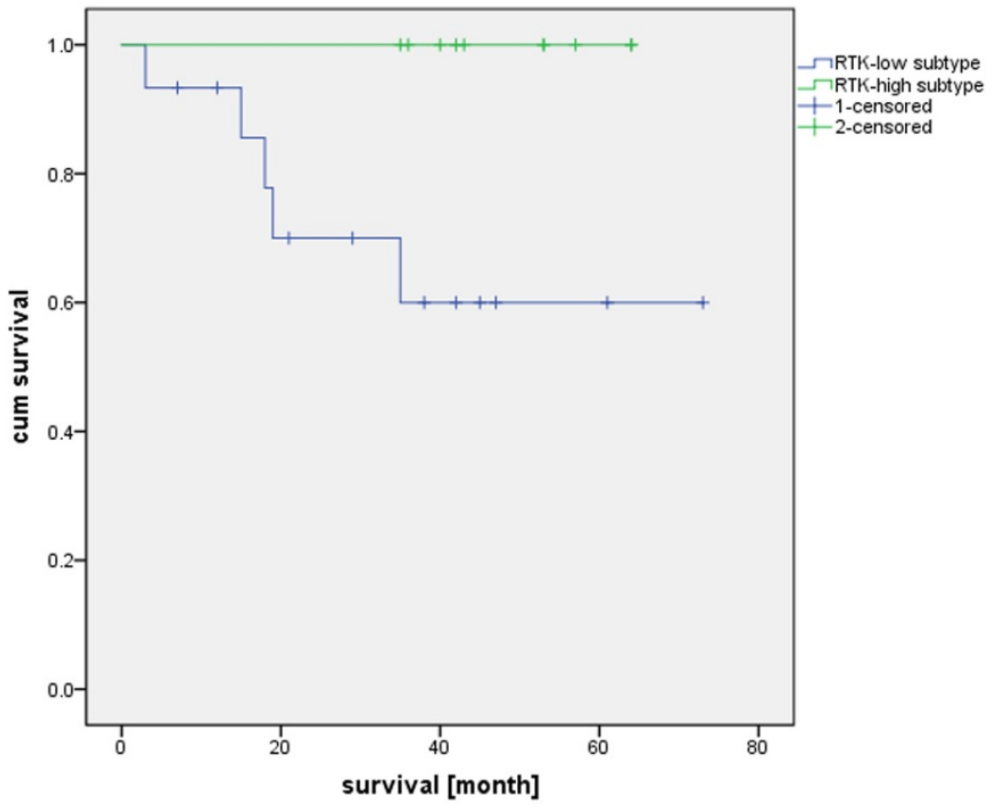

Figure 4. Overall survival of patients with RTK-high and RTK-low gene signature

work-up). For IGF-1R and EGFR the IHC findings were consistent with the gene expression data. Accordingly, differences in IGF-1R expression at both transcriptional and translational levels were found between the subtypes RTK-high and RTK-low (IHC: $p$ Mann-Whitney U test $=0.005$, RT-PCR: $p$ Mann-Whitney U test $<$ 0.001 ; $\mathrm{r}$ Spearman-Rho $=0.706, \mathrm{p} \leq 0.001)$. The expression profile of EGFR in both subtypes of triple-negative tumors could also be confirmed at the IHC level (IHC: $p$ Mann-Whitney U test $=0.377$, RT-PCR: $p$ $\mathrm{t}_{\text {-test }}=0.257 ; \mathrm{r}_{\text {Spearman-Rho }}=0.311, \mathrm{p}=0.100$ ).

As shown in Table 1, each case in the study group exhibited a triple negative phenotype according to established histopathological criteria. However, there were highly significant differences in the gene expression profiles for both ER ( $\mathrm{p}_{\text {Mann-Whitney }}$ $\mathrm{U}$ test $=0.001)$ and PR ( $\mathrm{p}$ Mann-Whitney $\mathrm{U}$ test $\leq 0.001)$ amongst the triple-negative tumors in relation to the postulated subtypes RTK-high and RTK-low. As with the IHC findings, no significant difference was found for HER2 at the transcriptional level between the postulated subtypes RTK-high and RTK-low ( $\mathrm{p}_{\text {t-test }}=$ 0.794; $\mathrm{r}$ Spearman-Rho $=-0.087, \mathrm{p}=0.655$ ).

\section{Immunohistochemical characterization of basal-like subtypes}

In relation to the much debated question as to the extent to which triple-negative tumors correspond to the basal-like breast cancer subtype, and to determine to what extent one of the two triple-negative subtypes defined in the study correlates with the basal-like subtype, IHC staining for the basal-like tumor associated biomarker CK5/ 6 and EGFR was performed. The IHC analyses revealed a basal-like phenotype within $79 \%$ of the samples. Statistical analysis did show no significant difference between the triple-negative subtypes defined in this study on the basis of gene expression analyses and the basal-like and non-basal-like TNBC subtypes ( $\mathrm{p}$ Pearson Chi-Square test $=0.080)$.

\section{Comparison of clinical parameters and genetic expression of tumor-related biomarkers}

By means of multivariate analysis the variables nodal status, age, tumor size and angiosis carcinomatosa were tested for a possible relationship to biomarker expression and subtype (RTK-high and RTK-low). The Chi-square test and Mann-Whitney $U$ test failed to show a significant relationship between any of the clinicopathological parameters and the subtypes.

T-test for equality of means and Levene's test for equality of variances demonstrated the statistic independence of tumor samples with adjuvant and neoadjuvant chemotherapeutic treatment in context to the RTK gene-expression analyses (Table 6). None of the changes was significant after Bonferroni correction.

\section{Discussion}

\section{Characterization and subtyping of triple-negative tumors based on therapeutically relevant $R T K$}

The search for individualized treatment strategies in recent years has revealed biomarkers with predictive relevance for a large number of malignancies. This information has been applied to the development of individualized tumor therapies. The various RTK are of special importance here, particularly representatives of the EGF and IGF receptor families which, in the case of overexpression, represent target proteins for individualized tumor therapy for an increasing number of tumor entities.

In the case of breast cancer, too, gene expression data and IHC findings have characterized patients for whom target-specific therapy is suitable. A number of clinical trials are concerned with the question as to what extent inhibition of EGFR or IGF-1R by specific monoclonal antibodies or RTKI can benefit patients with advanced breast cancer [35, 36, 37, 38, 39]. The combination of chemotherapy with EGFR inhibition appears so far to have mixed results. Adequate results of an individual therapeutic IGF-1R inhibition are not yet available. 
Table 6. Statistic independence of tumor samples with adjuvant and neoadjuvant chemotherapeutic treatment in context to the RTK gene-expression analyses

\begin{tabular}{|c|c|c|c|c|c|c|c|}
\hline Biomarker & $\begin{array}{l}\text { Fold Change } \\
\text { (neoadjuvant versus adjuvant) }\end{array}$ & p-value (Welch) & p-value (Levene) & Biomarker & $\begin{array}{l}\text { Fold Change } \\
\text { (neoadjuvant versus adjuvant) }\end{array}$ & p-value (Welch) & p-value (Levene) \\
\hline PR & -1.847 & 0.269 & 0.212 & PI3K_R1iso & -1.589 & 0.078 & 0.607 \\
\hline ER & -1.145 & 0.755 & 0.254 & AKT_com & 1.176 & 0.325 & 0.478 \\
\hline EGFR & -1.022 & 0.960 & 0.158 & SRC_com & -0.114 & 0.511 & 0.780 \\
\hline HER2 & 1.135 & 0.393 & 0.834 & SCUBE2 & -0.135 & 0.515 & 0.222 \\
\hline Her3 & -1.009 & 0.970 & 0.777 & BCL2 & -1.712 & 0.081 & 0.915 \\
\hline Her4 & -1.170 & 0.804 & 0.376 & IGF-1R & -1.454 & 0.390 & 0.690 \\
\hline TGF_alpha & -1.079 & 0.817 & 0.943 & IGF-2R & -1.403 & 0.090 & 0.250 \\
\hline EGF & -2.634 & 0.150 & 0.604 & IGF1 & -1.557 & 0.234 & 0.477 \\
\hline B_Cellulin & -1.125 & 0.826 & 0.279 & IGF2 & -1.297 & 0.729 & 0.605 \\
\hline Amphiregulin & -1.945 & 0.259 & 0.482 & HNF3-alpha & -1.842 & 0.318 & 0.046 \\
\hline HB_EGF & 1.183 & 0.573 & 0.924 & Survivin com & -1.600 & 0.117 & 0.765 \\
\hline NRG_com & -1.506 & 0.531 & 0.962 & TRIP 13 & 1.044 & 0.794 & 0.256 \\
\hline NRG_2 & 0.140 & 0.348 & 0.153 & CyclinB1 & -1.159 & 0.386 & 0.151 \\
\hline PTEN & -1.546 & 0.102 & 0.881 & Ki-67 & 1.037 & 0.840 & 0.161 \\
\hline PI3K_CA & -1.242 & 0.385 & 0.274 & LEF1 & -1.855 & 0.041 & 0.973 \\
\hline PI3K_R1com & -1.558 & 0.082 & 0.233 & & & & \\
\hline
\end{tabular}

At the same time, it has been shown in recent years that despite the importance of single tumor-relevant biomarkers, the neoplastic behaviour of tumors is influenced by numerous interactions in downstream signaling. The crosstalk of various overexpressed RTK, hormonal transcriptional regulation (ER) or mutational, constitutive activation in downstream signaling (EGFR, KRAS) represent multifactorial mechanisms involved in tumor growth. Because of the complexity of the situation, more recent clinical trials have focused increasingly on combination treatment strategies in narrowly defined patient groups. The characterization of breast tumors using a panel of tumor-specific surrogate markers not only contributes to a better understanding of functional mechanisms involved in tumor differentiation, growth and invasion, but also represents a basis for new combination treatment strategies. In addition, exact subtyping of breast cancer cases such as luminal A, luminal B, normal breast-like, ERBB2-overexpressing and basal-like molecular subtypes of breast cancer is still used for optimal patient selection in clinical trials.

In the study presented, we examined gene expression levels of various RTK and related biomarkers in TNBC without individualized treatment options with the aim of defining heterogeneous expression patterns and possible TNBC subtypes. Hierarchical cluster analysis was able to identify a panel of biomarkers that allows two distinct subtypes of TNBC to be distinguished. Further analysis of the genetic cluster emphasizes in particular the significance of the two RTK IGF-1R and HER4, as well as the hormone receptors ER and PR. Cases with overexpression of these markers described above (RTK-high) represent a potentially interesting group for clinical investigations. In addition, the possibility to identify those breast cancer patients who are potential responders for personalized medicine targeting IGF-1R or ER is of particular significance.

\section{Differentiation of triple-negative tumors into basal-like positive and negative subtypes}

Several microarray-based studies have demonstrated a differentiation of breast cancer into at least 5 different subtypes [40, 41, 42, 43]. Selected gene sets for around 500 biomarkers enable subtyping of breast carcinoma into luminal A, luminal B, normal breast-like, ERBB2-overexpressing and basal-like tumors. Tumors with the basal-like phenotype which are characterized by, among other features, by the absence of IHC ER and HER2 expression, are of particular clinical interest. Scientific studies employing IHC techniques and gene expression analysis have shown that up to $15 \%$ of all breast cancers exhibit the basal-like subtype [32, 33]. Numerous studies have attempted to equate triple-negative tumors with basal-like breast cancer, and indeed an accumulation of the basal-like phenotype amongst triple-negative tumors has been documented. Nevertheless, it has been shown that the two are not identical with each other [44]. Comparative analysis has shown that, although triple-negative tumors (immunohistochemically defined) often show a basal-like phenotype, and tumors diagnosed as basal-like breast cancer (on the basis of gene expression) are predominantly triple-negative, the two categories are up to $30 \%$ discordant $[19,45]$.

Methods for the identification of the basal-like phenotype rely mainly on the detection of expression of proliferation-specific biomarkers by gene expression analysis and immuno-histochemical techniques. Our study utilized IHC staining for CK $5 / 6$. Eighty-one percent of the tumor samples were found to express a basal-like phenotype, a prevalence consistent with figures quoted in the literature. 
In our study, comparison of the subtypes RTK-high and RTK-low and the group classed as CK $5 / 6$ positive revealed no significant correlation. Therefore, on the basis of our findings it can be assumed that this is a newly defined and independent gene expression signature that permits further subtyping of TNBC into RTK-high and RTK-low genotypes.

\section{Gene expression of ER in triple-negative breast cancer}

IHC analysis of the hormone receptor status, is one of the standardized clinical investigations used in the diagnostic work-up of patients with breast cancer for treatment prediction. Selection of the cases for our study was based upon the findings of routine histopathological investigations. By definition, all exhibited a negative ( $<1 \%)$ IHC score for ER and PR. By contrast, our gene expression analysis revealed over-expression of ER and PR in some cases, there being a highly significant difference between the subtypes RTK-high and RTK-low. The discrepancy may be due to the increased sensitivity of RT-PCR. Differences in regulation of protein expression at the translational level are also conceivable.

The results of correlation analysis pertaining to IGF-1R, HER4 and ER in tumors with the RTK-high genotype suggest that these receptors interact with each other through regulatory mechanisms. Hence, further in-vivo investigations, especially into the potential of new immunotherapeutic approaches using combined immunotherapeutic approaches in TNBC patients with the RTK-high genotype, would be of great interest $[46,47,48]$.

\section{Crosstalk between IGF-1R and ER signaling}

Recent studies have shown that there is crosstalk in cell signaling between RTK and ER. Various different trans-activating mechanisms with stimulating effects on the downstream signaling of adjacent RTK and ER were generally found $[49,50]$.

IGF-1R is one of the most important representatives of the IGF receptor family and, like $E R$, is associated with neoplastic properties when overexpressed [51, 52, 53].

In our study we found increased expression of IGF-1R and ER in TNBC of the RTK-high subtype. In addition, strong correlation between IGF-1R and ER was seen, which suggests functional interaction between the two receptors. As shown in recent studies, IGF-1R and ER are able to trans-activate each other and lead to activation or enhancement of downstream signaling [52]. In addition, in vivo experiments have demonstrated their combined significance in tumorigenesis [54].
In studies of IGF-1R-mediated ER activation [55, 56 , 57] it has been shown that tyrosine kinases involved in IGF-1R downstream signaling, such as MAPK, RSK and AKT, are able to phosphorylate and activate ER at the AF-1 domain (Serin118, Serin167). Furthermore, in vivo experiments showed that IGF-1 is able to increase the expression of $\mathrm{PR}$, an estrogen response element.

In addition to IGF-1R-mediated ER phosphorylation and activation, effects of ER on IGF-1R signaling have also been demonstrated [47]. ER is able, by means of various different regulatory mechanisms, to impact on IGF-1R downstream signaling. Studies have shown that anti-estrogens like tamoxifen can inhibit IGF-mediated growth [58]. Thus, it has been demonstrated that estrogens are able to regulate IGF-1R at the transcriptional level and to transactivate IGF-1R by phosphorylation. ER belongs to the steroid hormone receptor family and functions primarily as a transcription factor. IGF-1R is one of the most important estrogen response elements [58].

Besides the ability of ER to inactivate inhibitory elements of IGFR signaling, such as IGFBP-3, binding of ER to Shc with subsequent phosphorylation of MAPK have also been described. In addition to the singular importance of hormone receptors as a therapeutic target, several studies have demonstrated the existence of complex regulatory mechanisms and interactions in downstream signaling between ER/PR and growth factors [59, 60]. Several clinical trials concerning the combined use of IGF-1R and ER-inhibitors are under investigation [61, 62].

\section{Crosstalk between HER4 and ER signaling}

The c-erbB-4 gene (HER4) belongs to the EGFR family. Little is known about the functional significance of HER4 in the pathogenesis of breast cancer. In normal breast tissue, its functions include the differentiation of myocardial cells and mammary epithelial cells and the development of the central nervous system [63, 64]. Compared to the most important representatives of the EGFR family, EGFR and HER2, the prognostic impact of HER4 expression in breast cancer are basically unclear and controversially discussed $[65,66]$. Unlike the remaining members of the EGF receptor family, which act primarily as mitogenic effectors in breast cancer cells, HER4 appears to have a large number of different functions in normal and neoplastic breast tissue. With regard to the overexpression of HER4, experimental studies have shown both oncogenic and tumor suppressive functions [67, 68]. Other investigations have also shown that HER4 is generally associated with differentiated and less aggressive tumors, and that it correlates with a better prognosis 
and a longer disease-free interval [66, 69, 70]. In addition, it has been shown that breast cancer patients who exhibit coexpression of HER4 and ER have a lower recurrence rate [71] and a higher survival rate [72] than those who express ER alone.

Interestingly, several in vivo and in vitro studies have shown a strong correlation and crosstalk between HER4 and ER expression in breast cancer [68, $73,74]$. Intense efforts to elucidate mechanism of crosstalk between HER4 and ER could identify an autocrine HER4/ER signaling pathway where the factor HER4 intracellular domain (4ICD), a cleavage product of HER4, acts as transcriptional coregulator of $\operatorname{ER}[73,75,76,77,78]$.

Our results point to an increased rate of coexpression of HER4 and ER in triple-negative tumors with the RTK-high phenotype. The coexpression of HER4 and ER suggests the existence of crosstalk in downstream-signaling within defined subpopulations of breast cancer patients.

\section{ER / RTK crosstalk- development of intrinsic and acquired resistance}

Antihormonal therapy (tamoxifen, anastrozole) and HER2 inhibition (trastuzumab, lapatinib) represent important cornerstones in personalized therapy for the treatment of breast cancer in women with overexpression of the corresponding receptors. Further promising targets for individualized therapy the RTK EGFR and IGF-1R and targeting the PI3K-Akt pathway are currently subject of several studies and clinical trials [79, 80, 81, 82]. However, follow-up studies did show the development of resistance in some patients receiving antihormonal therapy and/or RTK inhibitors.

Studies have demonstrated intrinsic or acquired resistance to treatment with tamoxifen in $20-30 \%$ of hormone-receptor-positive breast cancer patients [83]. Although the mechanisms involved have not yet been clarified in detail, it appears that, in addition to coregulatory and epigenetic mechanisms, transactivating processes between RTK and ER could be responsible [80, 84]. Further investigations have revealed complex co-operation of genomic and non-genomic/fast ER signaling and their crosstalk with growth factor receptors, such as EGFR, HER2, HER4 or IGF-1R, in the development of endocrine resistance $[85,86,87]$. In brief, activated cytoplasmic and membrane-bound ER show the ability to stimulate RTK, either directly via adaptor proteins like Shc, or indirectly by nuclear ER through increased release of RTK-specific ligands, for example HB-EGF. Following subsequent activation of the downstream signaling kinases MAPK and AKT, phosphorylation and thus activation of nuclear ER occurs, which leads to increased gene expression, including the expression of RTK-related genes. Thus, innovative treatment strategies aim at inhibition of both RTK and ER.

Current indications for hormone receptor therapy are based on standardized IHC findings of routine diagnostic investigations. According to the histopathological guidelines and recommendations [28], antihormonal treatment is indicated when more than $1 \%$ of the tumor cells are ER and/or PR positive. Large overviews of randomized clinical trials have confirmed the therapeutic value of antihormonal treatment only in immunreactive ER-positive breast cancers.

Our investigations demonstrated a significant increase in ER gene expression level in a newly characterized subtype (RTK-high) of TNBC. In addition, correlation analysis suggests functional interaction between IGF-1R, HER4 and ER. It is not yet clear to what extent antihormonal therapy would be effective in tumors in which hormone receptor status is negative by immunohistochemistry but gene expression is elevated. However, on the basis of crosstalk between ER and RTK, it is conceivable that a combination of inhibition of both ER and corresponding RTK, like IGF-1R or HER4, could be of benefit.

\section{Conclusions}

RTK-associated gene expression profiles generated in this study revealed dichotomous differentiation within the triple-negative study group. IHC analysis verified this at the posttranslational level. Correlation analysis of the biomarkers investigated suggests a functional connection between IGF-1R, IGF-2R, HER4, PR and ER. Based on survival analysis the differentiation of triple-negative tumors with RTK-high and RTK-low gene signature seems to be from prognostic relevance.

\section{Abbreviations}

TNBC: Triple-negative breast cancers; ER: estrogen receptor; PR: progesterone receptors; IHC: immunohistochemical; RTK: receptor tyrosine kinase; RT-PCR: reverse transcription polymerase chain reaction; EGFR: epidermal growth factor receptor; RTKI: receptor tyrosine kinase inhibitors; IFF-1R: insulin-like growth factor receptor 1 ; $\mathrm{pCR}$ : pathological complete response; FFPET: formalin-fixed, paraffin-embedded tissue; HKG: housekeeping genes; 4ICD: HER4 intracellular domain.

\section{Acknowledgments}

We thank Annegret Schäfer of the IHC laboratory of the Institute of Pathology of the Ludwig 
Maximilians University of Munich for their dedication and contributions.

\section{Competing Interests}

The authors have declared that no competing interest exists.

\section{References}

1. American Cancer Society. Cancer Facts \& Figures 2014. https:// www.cancer.org/research/cancerfactsstatistics/cancerfactsfigures2014

2. Lakhani S, Ellis I, Schnitt S, et al. WHO Classification of Tumours of the Breast. 4rd ed. Lyon: IARC Press; 2012.

3. Ellis MJ, Perou CM. The Genomic Landscape of Breast Cancer as a Therapeutic Roadmap. Cancer Discovery. 2013; 3: 27-34.

4. Tessari A, Palmieri D, Di Cosimo S. Overview of diagnostic/targeted treatment combinations in personalized medicine for breast cancer patients. Pharmgenomics Pers Med. 2013; 7: 1-19.

5. Mayer IA, Abramson VG, Lehmann BD, et al. New strategies for triple-negative breast cancer-deciphering the heterogeneity. Clinical Cancer Research. 2014; 20: 782-790.

6. Kos Z, Dabbs DJ. Biomarker assessment and molecular testing for prognostication in breast cancer. Histopathology. 2016; 68: 70-85.

7. Yadav BS, Chanana P, Jhamb S. Biomarkers in triple negative breast cancer: A review. World J Clin Oncol. 2015; 6: 252-263.

8. Zhang JF, Liu J, Wang Y, et al. Novel therapeutic strategies for patients with triple-negative breast cancer. Onco Targets Ther. 2016; 21: 6519-6528.

9. Russell CA. Personalized medicine for breast cancer: it is a new day! Am J Surg. 2014; 207: 321-325.

10. Lehmann BD, Pietenpol JA. Identification and use of biomarkers in treatment strategies for triple-negative breast cancer subtypes. J Pathol. 2014; 232: $142-150$

11. Braicu C, Chiorean R, Irimie A, et al. Novel insight into triple-negative breast cancers, the emerging role of angiogenesis, and antiangiogenic therapy. Expert Rev Mol Med. 2016; 18: e18.

12. Ishitha G, Manipadam MT, Backianathan S, et al. Clinicopathological Study of Triple Negative Breast Cancers. J Clin Diagn Res. 2016; 10: EC05-EC09.

13. Lehmann BD, Jovanović $B$, Chen $X$, et al. Refinement of Triple-Negative Breast Cancer Molecular Subtypes: Implications for Neoadjuvant Chemotherapy Selection. PLoS One. 2016; 11: e0157368.

14. Engebraaten O, Vollan HK, Børresen-Dale AL. Triple-negative breast cancer and the need for new therapeutic targets. Am J Pathol. 2013; 183: 1064-1074.

15. Chiorean R, Braicu C, Berindan-Neagoe I. Another review on triple negative breast cancer. Are we on the right way towards the exit from the labyrinth? Breast. 2013; 22: 1026-1033.

16. Yau C, Esserman L, Moore DH, et al. A multigene predictor of metastatic outcome in early stage hormone receptor-negative and triple-negative breast cancer. Breast Cancer Research. 2010; 12: R85.

17. Chen $\mathrm{Xi}, \mathrm{Li} \mathrm{J}$, Gray $\mathrm{WH}$, et al. TNBC type: A Subtyping Tool for Triple-Negative Breast Cancer. Cancer Informatics. 2012; 11: 147-156.

18. Weisman PS, Ng CK, Brogi E, et al. Genetic alterations of triple negative breast cancer by targeted next-generation sequencing and correlation with tumor morphology. Mod Pathol. 2016; 29: 476-488.

19. Kreike B, van Kouwenhove M, Horlings H, et al. Gene expression profiling and histopathological characterization of triple-negative/basal-like breast carcinomas. Breast Cancer Research. 2007; 9: R65.

20. Sotiriou C, Soek-Ying N, McShane LM, et al. Breast cancer classification and prognosis based on gene expression profiles from a population-based study. PNAS. 2003; 100: 10393-10398.

21. Rakha EA, Ellis IO. Triple-negative/basal-like cancer: review. Pathology 2009;41:40-47.

22. Aifa S, Rebai A. ErbB antagonists patenting: "playing chess with cancer". Recent Pat Biotechnol. 2008; 2: 181-187.

23. Lurje G, Lenz HJ. EGFR signaling and drug discovery. Oncology. 2009; 77: 400-410

24. Irshad S, Ellis P, Tutt A. Molecular heterogeneity of triple-negative breast cancer and its clinical implications. Curr Opin Oncol. 2011; 23: 566-577.

25. Golan T,Javle M. Targeting the Insulin Growth Factor Pathway in Gastrointestinal Cancers. Oncology. 2011; 25: 518-526.

26. Gradishar WJ, Yardley DA, Layman R, et al. Clinical and Translational Results of a Phase II, Randomized Trial of an Anti-IGF-1R (Cixutumumab) in Women with Breast Cancer That Progressed on Endocrine Therapy. Clin Cancer Res. 2016; 22: 301-309.

27. Lin $\mathrm{EH}$, Lenz HJ, Saleh $\mathrm{MN}$, et al. A randomized, phase II study of the anti-insulin-like growth factor receptor type 1 (IGF-1R) monoclonal antibody robatumumab (SCH 717454) in patients with advanced colorectal cancer. Cancer Med. 2014 3. 988-997.

28. College of American Pathologists (CAP). Guideline Recommendations for Immunohistochemical Testing of Estrogen and Progesterone Receptors in Breast Cancer. https://www.ncbi.nlm.nih.gov/pmc/articles/PMC2931060/
29. Wolff AC, Hammond ME, Hicks DG, et al. Recommendations for human epidermal growth factor receptor 2 testing in breast cancer. American Society of Clinical Oncology. College of American Pathologists. Clinical practice guideline update. Arch Pathol Lab Med. 2014; 138: 241-256.

30. The Universal ProbeLibrary. https://www.lifescience.roche.com/products/ universal-probe-library

31. Pfaffl MW. A new mathematical model for relative quantification in real-time RT-PCR. Nucleic Acids Res. 2001; 29: e45.

32. Nielsen, TO, Hsu FD, Jensen K, et al. Immunohistochemical and Clinical Characterization of the Basal-Like Subtype of Invasive Breast Carcinoma. Clin. Cancer Res. 2004; 10: 5367-5374.

33. Dabbs DJ, Chivukula M, Carter G, et al. Basal phenotype of ductal carcinoma in situ: recognition and immunohistologic profile. Mod Pathol. 2006, 19: 1506-1511.

34. The R Project for Statistical Computing. https:// www.r-project.org

35. Arcaro A. Targeting the insulin-like growth factor-1 receptor in human cancer. Front Pharmacol. 2013; 4: 30-37.

36. Haisa M. The type 1 insulin-like growth factor receptor signalling system and targeted tyrosine kinase inhibition in cancer. J Int Med Res. 2013; 41: 253-264.

37. Gelmon K, Dent R, Mackey JR, et al. Targeting triple-negative breast cancer: optimising therapeutic outcomes. Ann Oncol. 2012: 23: 2223-2234.

38. Xue M, Cao X, Zhong Y, et al. Insulin-like Growth Factor-1 Receptor (IGF-1R) Kinase Inhibitors in Cancer Therapy: Advances and Perspectives. Curr Pharm Des. 2012; 18: 2901-2913.

39. Schwartz GK, Dickson MA, LoRusso PM, et al. Preclinical and first-in-human phase I studies of KW-2450, an oral tyrosine kinase inhibitor with insulin-like growth factor receptor-1/insulin receptor selectivity. Cancer Sci. 2016; 107: 499-506

40. Prat A, Perou CM. Deconstructing the molecular portraits of breast cancer. Molecular Oncology. 2011; 5: 5-23.

41. Perou CM, Sorlie T, Eisen MB, et al. Molecular portraits of human breast tumors. Nature. 2000; 406: 747-752.

42. Sorlie T, Perou CM, Tibshirani R, et al. Gene expression patterns of breast carcinomas distinguish tumor subclasses with clinical implications. Proc Natl Acad Sci U S A. 2001; 98: 10869-10874.

43. Sorlie T, Tibshirani R, Parker J, et al. Repeated observation of breast tumor subtypes in independent gene expression data sets. Proc Natl Acad Sci USA. 2003; 100: 8418-8423.

44. Irvin WJ Jr, Carey LA. What is triple-negative breast cancer? Eur J Cancer. 2008; 44: 2799-2805.

45. Bertucci F, Finetti P, Cervera N, et al. How basal are triple-negative breast cancers? Int J Cancer. 2008; 123: 236-240.

46. Chen HX, Sharon E. IGF-1R as an anti-cancer target--trials and tribulations. Chin J Cancer. 2013; 32: 242-252.

47. $\mathrm{Yu} Z$, Gao W, Jiang E, et al. Interaction between IGF-IR and ER induced by E2 and IGF-I. PLoS One. 2013; 8: e62642.

48. Chakraborty AK, Welsh A, Digiovanna MP. Co-targeting the insulin-like growth factor I receptor enhances growth-inhibitory and pro-apoptotic effects of anti-estrogens in human breast cancer cell lines. Breast Cancer Res Treat. 2010; 120: 327-335.

49. Zabransky DJ, Park BH. Estrogen Receptor and Receptor Tyrosine Kinase Signaling: Use of Combinatorial Hormone and Epidermal Growth Factor Receptor/Human Epidermal Growth Factor Receptor 2-Targeted Therapies for Breast Cancer. J Clin Oncol. 2014; 32: 1084-1086.

50. Heskamp S, Boerman OC, Molkenboer-Kuenen JD, et al. Upregulation of IGF-1R expression during neoadjuvant therapy predicts poor outcome in breast cancer patients. PLoS One. 2015; 10: e0117745.

51. Bartella V, De Marco P, Malaguarnera R, et al. New advances on the functional cross-talk between insulin-like growth factor-I and estrogen signaling in cancer. Cellular Signalling. 2012; 24: 1515-1521.

52. Liu C, Zhang Z, Tang H, et al. Crosstalk between IGF-1R and Other Tumor Promoting Pathways. Curr Pharm Des. 2014; 20: 2912-2921.

53. Christopoulos PF, Msaouel P, Koutsilieris M. The role of the insulin-like growth factor-1 system in breast cancer. Mol Cancer. 2015; 14: 43-56.

54. Bradley LM, Gierthy JF, Pentecost BT. Role of the insulin-like growth factor system on an estrogen-dependent cancer phenotype in the MCF-7 human breast cancer cell line. J Steroid Biochem Mol Biol. 2008; 109: 185-196.

55. Tremblay GB, Tremblay A, Copeland NG, et al. Cloning, chromosomal localization, and functional analysis of the murine estrogen receptor beta. Mol Endocrinol. 1997; 11: 353-365.

56. Simoncini T, Hafezi-Moghadam A, Brazil DP, et al. Interaction of oestrogen receptor with the regulatory subunit of phosphatidylinositol-3-OH kinase. Nature. 2000; 407: 538-541.

57. Becker MA, Ibrahim YH, Cui X, et al. The IGF pathway regulates ERa through a S6K1-dependent mechanism in breast cancer cells. Mol Endocrinol. 2011; 25: $516-528$

58. Fagan DH, Yee D. Crosstalk between IGF-1R and estrogen receptor signaling in breast cancer. J Mammary Gland Biol Neoplasia. 2008; 13: 423-429.

59. Ribeiro JR, Freiman RN. Estrogen signaling crosstalk: Implications for endocrine resistance in ovarian cancer. J Steroid Biochem Mol Biol. 2014; 143: 160-173.

60. Engels CC, de Glas NA, Sajet A, et al. The influence of insulin-like Growth Factor-1-Receptor expression and endocrine treatment on clinical outcome of postmenopausal hormone receptor positive breast cancer patients: A Dutch TEAM substudy analysis. Mol Oncol. 2016; 10: 509-516. 
61. Weroha SJ, Haluska P. IGF-1 receptor inhibitors in clinical trials--early lessons. J Mammary Gland Biol Neoplasia. 2008; 13: 471-483.

62. Fagan DH, Uselman RR, Sachdev D, et al. Acquired resistance to tamoxifen is associated with loss of the type I insulin-like growth factor receptor (IGF1R): implications for breast cancer treatment. Cancer Res. 2012; 72: 3372-3380.

63. Long W, Wagner K-U, Lloyd KCK. Impaired differentiation and lactational failure in ErbB4-deficient mammary glands identify ERBB4 as an obligate mediator of Stat5. Development. 2003; 130: 5257-5268.

64. Tidcombe H, Jackson-Fisher A, Mathers K, et al. Neural and mammary gland defects in ErbB4 knockout mice genetically rescued from embryonic lethality. Proc Natl Acad Sci USA. 2003; 100: 8281-8286.

65. Fujiwara S, Hung M, Yamamoto-Ibusuk CM, et al. The localization of HER4 intracellular domain and expression of its alternately-spliced isoforms have prognostic significance in ER+ HER2- breast cancer. Oncotarget. 2014; 5: 3919-3930.

66. Machleidt A, Buchholz S, Diermeier-Daucher S, et al. The prognostic value of Her4 receptor isoform expression in triple-negative and Her2 positive breast cancer patients. BMC Cancer. 2013; 13: 437-446.

67. Gullick WJ. c-erbB-4/HER4: friend or foe? J Pathol. 2003; 200: 279-281.

68. Junttila TT, Sundvall M, Lundin M, et al. Cleavable ErbB4 isoform in estrogen receptor-regulated growth of breast cancer cells. Cancer Res. 2005; 65: 1384-1393.

69. Portier BP, Minca EC, Wang Z, et al. HER4 expression status correlates with improved outcome in both neoadjuvant and adjuvant Trastuzumab treated invasive breast carcinoma. Oncotarget. 2013; 4: 1662-1672.

70. Wang J, Yin J, Yang Q, et al. Human epidermal growth factor receptor 4 (HER4) is a favorable prognostic marker of breast cancer: a systematic review and meta-analysis. Oncotarget. 2016; 7: 76693-76703.

71. Barnes NL, Khavari S, Boland GP, et al. Absence of HER4 expression predicts recurrence of ductal carcinoma in situ of the breast. Clin Cancer Res. 2005; 11: 2163-2168.

72. Witton CJ, Reeves JR, Going JJ, et al. Expression of the HER1-4 family of receptor tyrosine kinases in breast cancer. J Pathol. 2003; 200: 290-297.

73. Rokicki J, Das PM, Giltnane JM, et al. The ERalpha coactivator, HER4/4ICD, regulates progesterone receptor expression in normal and malignant breast epithelium. Mol Cancer. 2010; 9: 150-154.

74. Pawlowski V1, Révillion F, Hebbar M, et al. Prognostic value of the type I growth factor receptors in a large series of human primary breast cancers quantified with a real-time reverse transcription-polymerase chain reaction assay. Clin Cancer Res. 2000; 6: 4217-4225.

75. Thor AD, Edgerton SM, Jones FE. Subcellular localization of the HER4 intracellular domain, 4ICD, identifies distinct prognostic outcomes for breast cancer patients. Am J Pathol. 2009; 175: 1802-1809.

76. Jones FE. HER4 intracellular domain (4ICD) activity in the developing mammary gland and breast cancer. J Mammary Gland Biol Neoplasia. 2008; 13: $247-258$

77. Naresh A, Thor AD, Edgerton SM, et al. The HER4/4ICD estrogen receptor coactivator and BH3-only protein is an effector of tamoxifen-induced apoptosis. Cancer Res. 2008; 68: 6387-6395.

78. Zhu Y, Sullivan LL, Nair SS, et al. Coregulation of estrogen receptor by estrogen-inducible ERBB4/HER4 establishes a growth promoting autocrine signal in breast cancer. Cancer Res. 2006; 66: 7991-7998.

79. Nabholtz JM, Chalabi N, Radosevic-Robin N, et al. Multicentric neoadjuvant pilot Phase II study of cetuximab combined with docetaxel in operable triple negative breast cancer. Int J Cancer. 2016; 138: 2274-2280.

80. Engels CC, de Glas NA, Sajet A, et al. The influence of insulin-like Growth Factor-1-Receptor expression and endocrine treatment on clinical outcome of postmenopausal hormone receptor positive breast cancer patients: A Dutch TEAM substudy analysis. Mol Oncol. 2016; 10: 509-516.

81. Iams WT, Lovly CM. Molecular Pathways: Clinical Applications and Future Direction of Insulin-like Growth Factor-1 Receptor Pathway Blockade. Clin Cancer Res. 2015; 21: 4270-4277.

82. Massacesi C, Di Tomaso E, Urban P, et al. PI3K inhibitors as new cancer therapeutics: implications for clinical trial design. Onco Targets Ther. 2016; 9: 203-210.

83. Ali S, Rasool M, Chaoudhry H, et al. Molecular mechanisms and mode of tamoxifen resistance in breast cancer. Bioinformation. 2016; 12: 135-139.

84. Higgins MJ, Stearns V. Understanding resistance to tamoxifen in hormone receptor-positive breast cancer. Clinical Chemistry. 2009; 55: 1453-1455.

85. Arpino G, Wiechmann L, Osborne CK, et al. Crosstalk between the estrogen receptor and the HER tyrosine kinase receptor family: molecular mechanism and clinical implications for endocrine therapy resistance. Endocrine Reviews. 2009; 29: 217-233.

86. Koutras AK, Fountzilas G, Kalogeras KT, et al. The upgraded role of HER3 and HER4 receptors in breast cancer. Crit Rev Oncol Hematol. 2010; 74: 73-78.

87. Osborne CK, Schiff R. Mechanism of Endocrine Resistance in Breast Cancer. Annu Rev Med. 2011; 62: 233-247. 